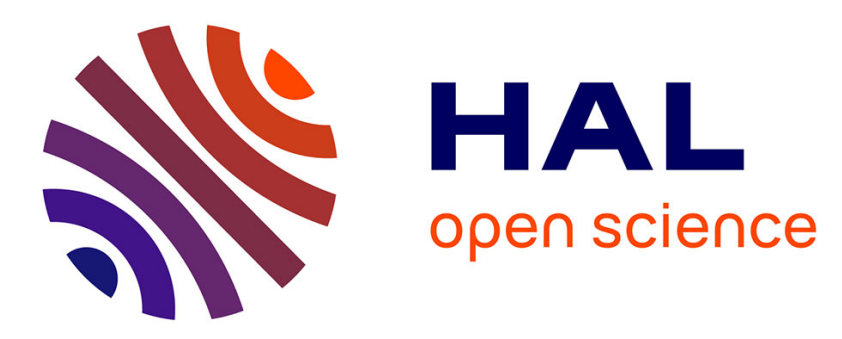

\title{
Wireless Network Architecture for Diagnosis and Monitoring Applications
}

Zeashan Hameed Khan, Denis Genon-Catalot, Jean-Marc Thiriet

\section{To cite this version:}

Zeashan Hameed Khan, Denis Genon-Catalot, Jean-Marc Thiriet. Wireless Network Architecture for Diagnosis and Monitoring Applications. MASAUM Journal of Computing, 2009, 1 (2), pp.318-325. hal-00423732

\section{HAL Id: hal-00423732 \\ https://hal.science/hal-00423732}

Submitted on 12 Oct 2009

HAL is a multi-disciplinary open access archive for the deposit and dissemination of scientific research documents, whether they are published or not. The documents may come from teaching and research institutions in France or abroad, or from public or private research centers.
L'archive ouverte pluridisciplinaire HAL, est destinée au dépôt et à la diffusion de documents scientifiques de niveau recherche, publiés ou non, émanant des établissements d'enseignement et de recherche français ou étrangers, des laboratoires publics ou privés. 


\title{
Wireless Network Architecture for Diagnosis and Monitoring Applications
}

\author{
Zeashan H. Khan, Student Member, IEEE, Denis G. Catalot and J.M. Thiriet, Member, IEEE
}

\begin{abstract}
This paper describes a distributed wireless network architecture for remote diagnosis and monitoring. Wind energy conversion system (WECS) is considered as the target application, where windmills are grouped into small clusters communicating with each other for the purpose of distributed diagnosis. The evaluation of this network is simulated to support effective communication needs required for fault detection and as a result to send an alarm or caution message to the remote monitoring station. However, as the sensitivity of application increases, strict requirements on availability, robustness, reliability and performance of network resources must be satisfied in order to meet industrial standards.
\end{abstract}

Index Terms - Condition monitoring, distributed diagnosis, heterogeneous wireless network, windmill farms.

\section{INTRODUCTION}

$\mathrm{W}$ IRELESS networks are best suited for remote management and teleoperation of complex industrial plants as they offer more flexibility, reduced costs, better power management, ease in maintenance, and quick deployment in distant hard-to-reach areas where installation of conventional wired infrastructure is not possible. Major applications for wireless networks in industry involve maintenance, security, monitoring, control, diagnosis etc as in [1], [2] and [3]. From unmanned aerial and ground vehicles, industrial machinery, chemical plants, traffic management over highways to remote under water vessels, the applications are vast and diverse. In traffic management and roadside safety, this also includes collision warning and avoidance on highways, platooning, time varying flow control, precedence for ambulance and police vehicles etc. Wireless networked communication connects the autonomous systems in two ways. First with in the cluster e.g. in case of multiple vehicles a local consensus is maintained for cooperative decisions.

This work is funded by the Higher Education Commission (Pakistan), in collaboration with SFERE (France) under OSS-06 program.

Zeashan Hameed Khan is with the Control Systems Department, GIPSA-lab, Grenoble, 38402, France. (Phone: +33476827159; fax: +33476826388; e-mail: zeashan-hameed.khan@ gipsa-lab.inpg.fr).

Denis Genon-Catalot is currently with LCIS, BP54 26902, Valence, France. (e-mail: denis.genon-catalot@iut-valence.fr).

Jean Marc Thiriet is with the Control Systems Department, GIPSA-lab, Grenoble, 38402, France. (e-mail: jean-marc.thiriet@ gipsa-lab.inpg.fr).

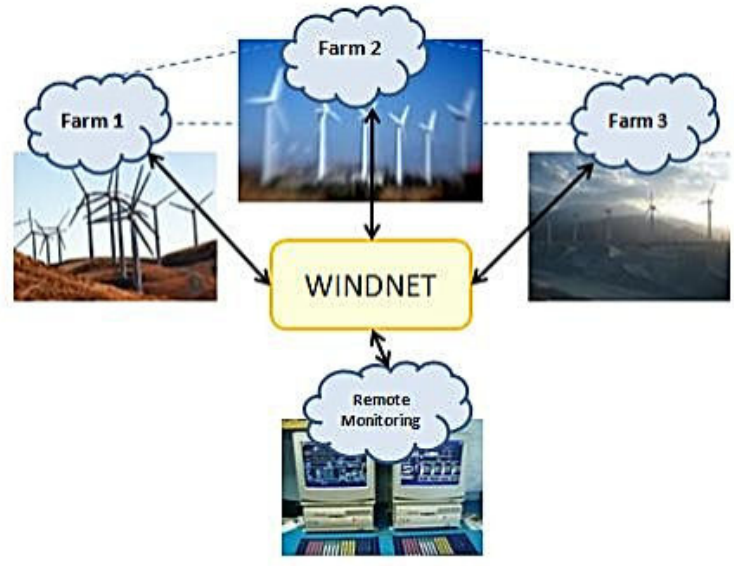

Fig. 1. Distributed Windmill Energy Generation System

Vehicle to vehicle communication (V2V) and formation control of multiple vehicles are some of the known examples as described in [4], [5] and [6]. On the other hand, if autonomous systems are controlled from outside via infrastructure, then this remote teleoperation becomes more complex. In such applications, long distance communication protocols are implemented with guaranteed service quality to ensure mission objectives. Examples include vehicle to infrastructure communication (V2I), teleoperated robotic arms with force feedback and camera feedback for remote surgery, lunar rover controlled by earth station etc. Ad hoc or self organizing networks have each node acting as a source and destination for data. In addition, it also performs the function of a router. If mobility is considered, then infrastructural aids can be added in the network architecture to avoid communication loss due to link breaks in the ad hoc network. Thus, hybrid network architecture involving infrastructure as well as ad hoc network can also be considered in such applications. Fault diagnosis is stressed in addition to control as there may be instants for unplanned scenarios or when backup redundancies are not sufficient for the required objectives, so an operator intervention may be necessary once the fault is diagnosed and identified. In a general context, the dependence of the control and diagnosis of industrial systems on physically connected communication infrastructure has been relaxed by the promising wireless technologies, ranging from wireless sensor networks to broadband and satellite networks [7]. In this paper, wireless network architecture is proposed for control, condition 
dependent monitoring and fault diagnosis of large scale distant windmill installations as shown in Fig. 1. Different supervision levels are defined to address the issue faced by wireless communication systems in delivering diagnosis data by minimizing the imperfections of wireless networks [8]. Thus it is a co-design problem in which the objective is to maximize the control and diagnosis quality that will be affected due to network quality of service (QoS) offered by the communication network. In addition, the complexity of the problem will be raised when different RF links would have to be used to satisfy the requirements of end to end network QoS which is further dependent on bandwidth, payload, delay, jitter, range, noise immunity, interference rejection and cost for communication [9].

\section{CONTEXT}

Diagnosis and Monitoring of industrial systems inherently requires a distributed approach, which offers some widely recognized advantages like the possibility of enhanced reliability due to component redundancy, improved performance by exploiting concurrency and better resource management and utilization through sharing [10]. A local performance measure thus plays an important role in distributed hierarchy. The goal of communication, at different levels, for monitoring and supervision of windmill farms, can be achieved with low cost wireless network architecture, taking into account the constraints of noise, interference, bandwidth limitations and security [11]. A compromise between requirements and limitations will result in a promising solution of the stated problem. A lot of research in distributed diagnosis has considered computer systems connected with a network, for example in [12], a Hierarchical Adaptive Distributed System-level Diagnosis algorithm is presented which allows every fault-free node to achieve diagnosis in maximum $\left(\log _{2} \mathrm{~N}\right)^{2}$ testing rounds. In [13], a diagnostic Algorithm HeartbeatComplete is presented, which offers bounded correctness in fullyconnected systems while simultaneously minimizing diagnostic latency, start-up time, and state holding time. In [14], a rule based diagnosis through a monitor system for diagnosis in large scale network protocols is discussed. While in [15], the authors reflect some experience in reliability and backup operation. Monitoring and diagnosis of autonomous systems is also getting popular. For example, in [16], an approach for distributed diagnosis of coupled mobile robots is presented. The information flow between the entities (sensors, actuators or process itself) connected to a network requires firm guarantees w.r.t time bounds (delay and jitter), availability and bandwidth allocation in order to exhibit control and monitoring performance. One approach to reduce the time delays for rapid supervision is to dedicate a part of distributed intelligence within the local cluster e.g. by utilizing wireless sensor networks with local data logging and processing for alarms and monitoring. However, communication needs in distributed applications are wide spread and the choice of network architecture is a critical decision.

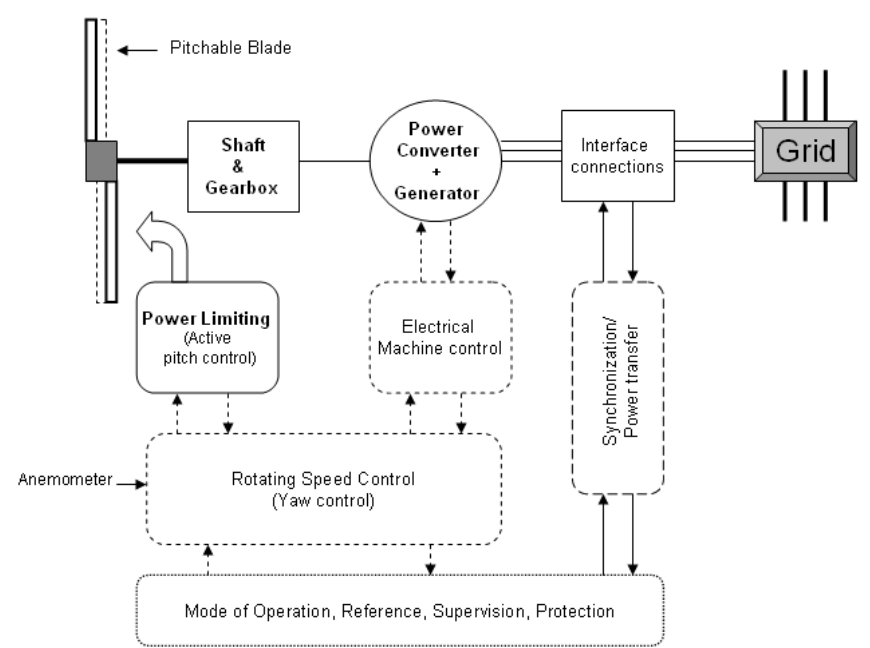

Fig. 2. Windmill Components and Subsystems

\section{WIND ENERGY CONVERSION SYSTEM}

Generating electricity from wind is widely seen as one of the most popular and cost effective way of green energy sources. Thus more and more windmill installations are being planned in most parts of the world for pollution free energy generation. Windmills simply act as transducers, converting the wind into either mechanical or electrical energy. If the operational efficiency of the windmill farm can be increased through active surveillance and fault diagnosis, then the need for expensive, polluting, power generators will be reduced. The control and diagnosis strategy will take into account the developing faults that may develop into failures.

Wind energy conversion systems (WECS) can be classified based on their position relative to ground (horizontal or vertical), number of rotor blades, drive type (direct or indirect), type of installed generator, type of control used etc. The details can be seen in [17]. For simplicity, a general purpose horizontal axis wind turbine is under consideration as shown in Fig. 2. The major parts shown in the Figure 2 are explained as follows:

\section{A. Major Subsystems}

1) Rotor: A wind energy conversion system essentially consists of an aerodynamic rotor with 2 to 3 blades, joined with a hub, cutting incoming wind (at an incident angle) and driving a generator. Light weight but strong designs are usually preferred in rotor manufacturing. Structural faults may occur due to cracks in one or more rotor blades or due to malfunction of speed control system.

2) Gearbox: This component is used for mechanical power transmission to the generator. Today, high performance gearboxes with gear ratios up to $1: 100$ and more are available [18]. Faults like misalignment with the generator, oil degradation, overheating during operation, mechanical failures and locking can cause over or under speed of the rotor.

3) Generator: The wind energy is thus converted into 
mechanical energy and then electrical power. Due to lubrication or cooling system failure, a generator can catch fire which could be destructive.

4) Nacelle: The electro-mechanical system of a wind turbine is housed in a closed nacelle. Their sizes are a compromise between strategy to reduce weight as well as to provide enough space for maintenance technicians working onboard. Nacelles may encounter a structural damage that may be fatal.

5) Yaw System: This is an optional system, used today in most of the windmills. An active yaw control system consists of a drive motor, electronics and further gearing to optimize windmill performance by changing relative position with the direction of wind. Locking and braking systems are also utilized for security measures. A fault in Yaw system will result in maneuver failure, which can degrade windmill performance.

6) Pitch System: The blade pitch control system (PCS) is mostly used in windmills today. The primary task of PCS is to adjust the blade pitch angle (normally $20^{\circ}$ to $25^{\circ}$ ) for controlling the power and speed of the rotor. The secondary task is to use it as aerodynamic brakes by pitching approximately to $90^{\circ}$. A fault in pitch system can not only degrade its performance but can also be dangerous due to over speed or loss of power control.

\section{B. Supervision and Protection}

This is one of the most important subsystem of all modern wind turbines, which does the purpose of protection and emergency stop in case of turbine over speed, lightening strikes, Blade frost (icing) and other mechanical failures. It recognizes local faults by using sensor data, to act as soon as possible to avoid complete failure. In addition, some of the data is shared with other wind turbines and the farm supervisor for health status ranking of each turbine that will finally end up predict the number of remaining service hours. The time critical nature of these tasks demand a dependable communication system with bounded delay through which faults can be reported and emergency actions can be taken. The frequency of this feedback determines the minimum end to end bandwidth and data rate demand expected from the wireless network. A 'heartbeat' status reporting requirement for each block may be different depending upon its importance and mode of operation.

\section{Production Optimization}

The objectives of communication in the windmill farm are not only to detect faults and perform teleoperation but also to maximize the production of electricity. Aerodynamic array efficiency is important to minimize the aerodynamic interference of windmills installed in a geometric array. The distance between windmills installed in an array is found to be 8 to 10 times the rotor diameter in the prevailing wind direction and 3 to 5 times rotor diameter across the main wind direction [18]. These distances must be taken into account while choosing the communication protocol in the wind farm to communicate between the windmills. The electrical power output PE is a function of wind speed $\mathrm{v}$, air density $\rho$, turbine power characteristics $c_{p}$ and the area of the rotor $A_{r}$ as [19]:

$$
P_{E}=0.5 \rho v^{3} c_{p} A_{r}
$$

Where, $\mathrm{cp}$ is the turbine power characteristic coefficient which is a multiple of rotor characteristics $c_{p r}$ and the efficiency $\eta$ transferred through mechanical-electrical coupling. The rotor power curves $c_{p r}$ exhibit how power coefficient varies with the tip speed ratio of the windmill rotor. The $c_{p r}-v$ curves provide the rotor dynamics due to wind variations. The speed control of the windmill farm is one of the global objectives which could be a collaborative task by exchanging this information among each individual windmill controller.

\section{Diagnosis and Monitoring Needs}

The condition monitoring in the windmill systems is aimed to accurately diagnose the condition of the equipment during operation. This will not only help in predictive maintenance but also in the health monitoring of each system. The fault diagnosis in industrial systems stems from analysis of some key signals from sensors. Frequency response analysis is most often used for fault identification related to windings of electrical machines [20]. This data is analyzed at the first place by the onboard embedded system. The limitations of embedded diagnosis include the limited computational power available, cost and complexity.

1) Requirements: The requirements for the monitoring procedure includes the choice of subsystems most often affected, frequency of analysis, type and number of sensors, redundant components for critical parts etc. In addition, the distribution of diagnosis tasks between the onboard and offboard components is also important to consider.

2) Sensor Suite: Diverse sensor suits are available in today's modern installations. The most common type of sensor is the accelerometer, needed to detect structural modes and their shift due to fault. Some systems use acoustic sensors as well to detect unusual sounds that can caution a developing anomaly. Other sensors include, oil contamination detector, temperature and humidity sensors. In addition, current and voltage measurements are also monitored for the expected system outcomes. Ice detection is important in areas with extensive snowfall. Video cameras have also been mounted on some of the systems to detect bird hit and avoid the destructive consequences. In general, the information sources may be voice, video and data, which will be analyzed and may need to be sent over a distant communication network. 


\section{Proposition of A General Architecture}

The generalized network based communication architecture can be visualized at various levels, with every level having its own constraints (end to end QoS requirements, time delays, network traffic, packet bursts, multi path effects, Electromagnetic interference (EMI) etc). We group a cluster of windmills installed within a closer distance and call it a Windmill Farm. While a group of windmill farms is jointly labeled as a Windmill Park. This architecture is shown in Fig. 3.

\section{A. Level 1: Windmill Based Network: (WBN)}

The WBN is composed of sensors, actuators and controllers connected to each subsystem. The data acquisition of various components of a windmill is performed by a local supervisor node. The windmill based network is essentially a low power wireless mesh network. However, classical field buses have also been used in such systems [21]. Each sub-component of windmill e.g. gear box, generator, nacelle etc have sensors attached to them, which send data to the local supervisor node through a wireless network. The parameters of interest include vibration data of gear box and generator, health indicator signals of electrical subsystems, generated voltage, status of AC/DC/AC block, Gear box oil temperature etc.

\section{B. Level 2: Farm Based Network (FBN)}

This network is responsible for the communication within the various windmills of a farm for control and diagnosis purpose. The exchange of data is between individual windmills as well as with the farm supervisor node, which is responsible for the data management and diagnosis decision at the farm level. The information of interest may include the wind direction relative to windmill farm, voltage generated by each individual turbine and condition monitoring parameters to decide and optimize farm productivity.

\section{Level 3: Inter-Farm (Park) Based Network (PBN)}

The communication task between various distributed windmill farms of a region is taken care by an inter-farm network. Fig. 3 shows a PBN composed of many FBNs. One of the tasks for this level of communication would be as simple as to exchange diagnosis information to ensure interrupt free voltage stabilization to the grid with optimized use of windmills with changing electrical loads and wind conditions.

\section{Level 4: Distant Supervision Network (DSN)}

This level of communication network will involve long distance communication between windmill parks that are distributed usually far away (especially for the case of offshore windmills) and the supervisor at the diagnosis center. The end to end delays involved are crucial at this level.

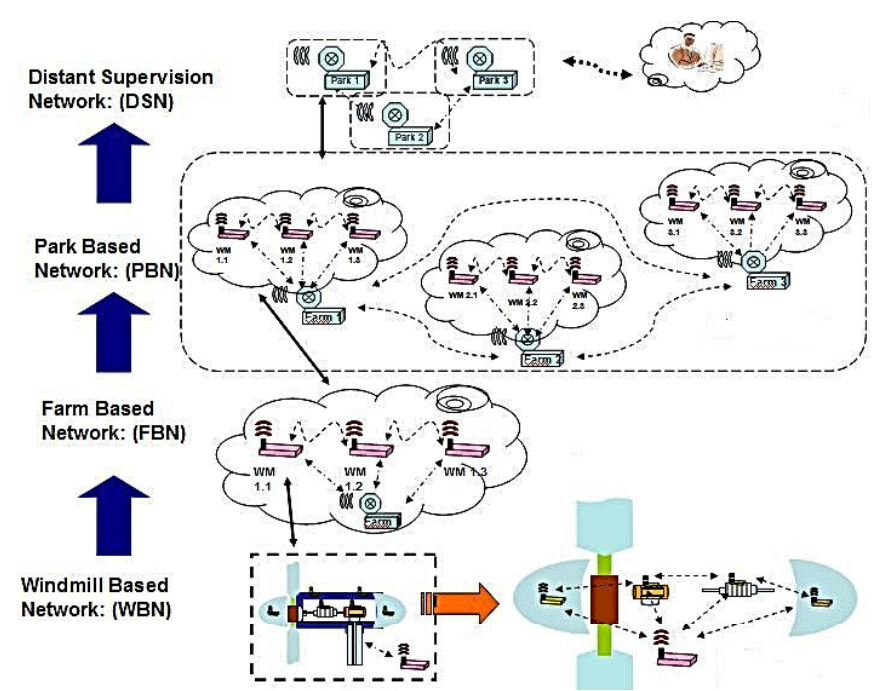

Fig. 3. General hierarchical Network architecture

\section{DistRIBUTED DiAgnosis}

The distributed diagnosis concept of windmill farms is based on the network resources available at different levels. Typically, faults in windmill subsystems develop slowly e.g. contamination of oil in the gear box, rise in temperature of generator windings, excessive nacelle vibration due to structural faults etc. So the measurement data from sensors related to condition monitoring may not be time critical. The measurement data can be processed and analyzed at different levels of the network. The amount of data to be transferred can be reduced sufficiently by emphasizing local analysis. The measurement parameters traditionally used in vibration measurements are displacement $(\mathrm{m})$, velocity $(\mathrm{mm} / \mathrm{s})$ and acceleration (m/s2). ISO 10816-3 classifies vibration velocity amplitudes in the frequency range of 10 to $1000 \mathrm{~Hz}$ as a function of turbine output [22]. Impact-like phenomena, such as bearing and gear faults, friction and insufficient lubrication, can be studied by means of acceleration measurements more effectively than using velocity measurements. For wind turbines, gear manufacturers have defined typical frequency range of 10 $\mathrm{Hz}$ to $100 \mathrm{~Hz}$. The frequency information of vibrations is continuously monitored at a higher rate and a local check is maintained on permissible amplitude of the vibration. In addition, sensor faults of individual windmills can be detected and a collaborative monitoring system at the farm based network (FBN) will diagnose and improve farm based electricity generation. For example, in case of anemometer fault at one windmill, farm supervisor node can estimate and still maneuver the faulty windmill for optimum wind direction based on data received from other anemometers of the farm windmills.

\section{PRotocol Choice}

For simplicity only 2-tier architecture composed of FBN and PBN is discussed here. The tier 1 comprises of each windmill collaborating with in the FBN. The second tier links the PBN with a node in FBN. The choice of protocol in each tier is crucial. 


\section{A. Tier 1 Protocol}

The tier 1 protocol has to deal with in short distances and data rate requirements and availability is time critical. In applications incorporating structural health monitoring, medical surveillance and habitat monitoring, LR-WPANs (e.g. 802.15.4) are preferred due to their small size and low power consumption. While for industrial applications, Wireless HART is very popular which uses channel black listing to avoid interference, offers robust communication and guarantees time slots of $10 \mathrm{~ms}$. However, if high data rate, security and bandwidth are required, WLAN (802.11) with some variations is the preferred choice for infrastructure based networks. LRWPAN offers low data rate and at the same time requires CFP (collision free period) management by the PAN coordinator for real time transmission through guaranteed time slots (GTS). Another choice is 6lowpan which is based on 802.15.4 but support IPv6 [23]. In this way, end to end IP compatibility can be ensured if the second tier is also IP compliant, despite the difference of MAC protocol. The 6lowpan working group for IPV6 over IEEE 802.15.4 have proposed its recent draft for routing protocols and network management. However the draft is not finalized yet. The ease of using 6lowpan is that existing infrastructure can be used due to pervasive nature of IP. There is a large address space available to assign to every node with out the need of network address translation (NAT). This offers a simple gateway architecture with end to end IP support.

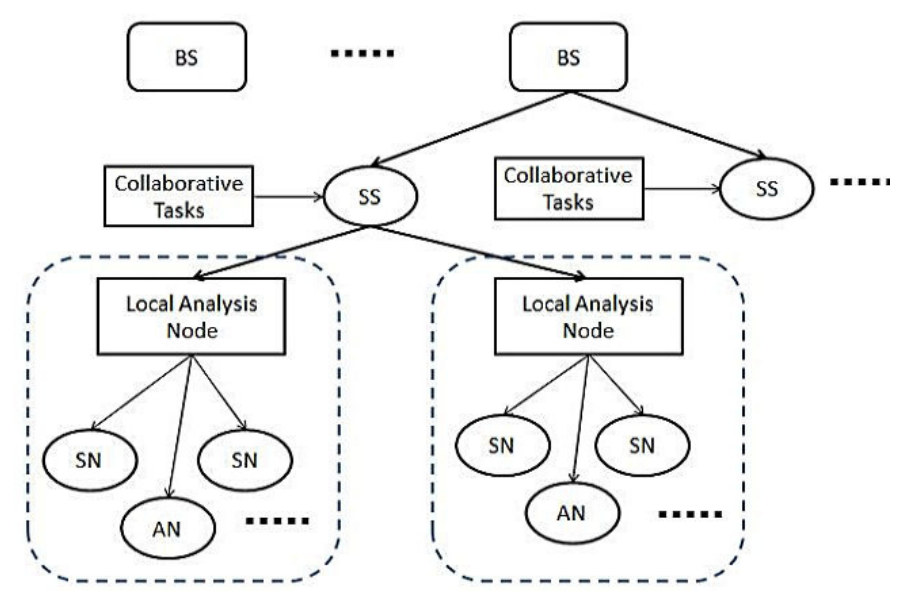

Fig. 4. Diagnosis with the 2 tier architecture $(\mathrm{SN}=$ sensor node, $\mathrm{AN}=$ Actuator node, $\mathrm{SS}=$ Subscriber Station, BS = Base station)

\section{B. Tier 2 Protocol}

The tier 2 needs a long range communication protocol with extensive QoS support. Traditional mobile communication protocols e.g. GPRS offers QoS classes based on priority, reliability, delay and bandwidth required for the user applications. However, more rigid IP based QoS policies are proposed for GPRS/UMTS backbone network. WiMAX is one of the strong candidates for tier 2 protocol with several important factors that distinguish WiMAX from other wireless technologies being an IP- based network, relatively simple network topology than cellular networks, takes less equipment and less time to set up infrastructure with lower equipment and full mobility support for users. WiMAX has both the options of frequency and time division duplexing (FDD and TDD); however, FDD does not provide the optimum use of resources. Whereas, with TDD, operators are capable of adjusting downlink and uplink ratios based on their service needs in the networks [2].

\section{QOS IN 2 TIER ARCHITECTURE}

Quality of Service (QoS) is considered as a combination of some key network characteristics e.g. Bandwidth, latency, jitter, packet loss and reliability that determines if a wireless technology can successfully deliver high value services (e.g. voice, data, video). On the other hand, there are applications which involve ensuring QoS at certain time interval for sending critical information (e.g. event based alarms) in real time from one network node to another. In order to achieve this target we need to evaluate QoS parameters and mechanisms available in all subsequent networks involved in the communication path.

A simple case of 2 tier network is considered here as shown in Fig. 4. A sensor network utilizing 802.11 in the first tier while 802.16 (WiMAX) is considered in the second tier. The network architecture is analyzed to evaluate the suitability for real time data communication for control and collaboration over wireless network involving large distances. The generalized QoS consideration ensures that those packets sent through CFP on 802.11 should be allocated greater priority (e.g. rtPS) in WiMAX network to make real time message transmission possible. However for full-IP or end to end IP networks are considered, than the QoS will not be dependent on different MAC layers. Instead IP QoS parameters can be used for end to end performance.

TABLE II QOS OPTIONS IN WLAN

\begin{tabular}{ccc}
\hline \hline WLAN version & Management & Access Mode \\
\hline $802.11 \mathrm{~b} / \mathrm{g}$ & Access Point (AP) & DCF/PCF \\
\hline $802.11 \mathrm{e}$ & Hybrid coordinator (HC) & $\begin{array}{c}\mathrm{HCF} \\
(\mathrm{EDCA} / \mathrm{HCCA})\end{array}$ \\
\hline \hline
\end{tabular}

A. QoS provisions in $802.11 \mathrm{~b} / \mathrm{g}$

The basic WLAN is critical next generation network (NGN) but has least developed QoS mechanisms, due to its contention based medium access (CSMA/CA) mechanisms. In addition, the existing WLAN QoS schemes implement QoS on individual networks independently. Since IEEE 802.11 is connectionless, a node that sends a transmission cannot detect the state of the network a priori. The MAC layer defines two different access methods, the 'asynchronous data transmission' mode or distributed coordination function (DCF) which is used for random 
access 'best-effort' traffic. The other one is the point coordination function (PCF) for time constrained traffic which uses contention free periods (CFP). The IEEE 802.11 standard specifies that the CSMA/CA with RTS/CTS and Hybrid coordination function (HCF) are optional. HCF is based on a polling approach as well as differentiated transmission by assigning different waiting periods to nodes with different real-time data transmission constraints. CFP is the HCF period and contention period (CP) is the duration of contention. Beacons or control frames are used to start superframes in CFP. Traffic on these network technologies, however, is subject to delay, making bandwidth availability and delivery time difficult to predict. Although high-priority traffic typically arrives at its destination before lower-priority traffic as per demands of time-flexible telecom applications, it cannot be guaranteed to arrive within a specified time needed in time critical applications. Despite the fact that QoS is more difficult to implement on connectionless networks, there is growing interest in developing QoS for IP-based networks. It offers a very low latency across the wireless span. In 802.11e there are 2 co-ordination functions in Hybrid Coordination Function (HCF). The first coordination function is the Enhanced Distributed Channel access (EDCA), which is a QoS enabled version of DCF and the HCF Controlled Channel Access (HCCA), which is similar to the PCF.

\section{B. QoS provisions in 802.11 e}

The IEEE 802.11e is a QoS oriented version of 802.11 protocol and it supports QoS for a wide variety of applications including voice, video, data etc. The hybrid Coordination function (HCF) is an enhancement in 802.11e which is further divided into two categories as in the basic protocol. The new traffic categories are HCF controlled channel access (HCCA) for contention free access and Enhanced Distributed Channel Access (EDCA) for contention based access. The idea is to time slice the prioritized flows in polling as well as to use class based differentiation. Thus, EDCA define access categories for prioritized flows while in HCCA, a hybrid coordinator (HC) allocates transmission opportunities (TXOPs) to nodes by polling for the contention-free transfers of data, based on QoS policies [9]. These traffic stream queues offer strict QoS as compared to access categories in EDCA.

1) EDCA: In 802.11e EDCA mode, the priority information is passed on to the MAC layer from the higher layers for priority based transmit opportunity (TXOP). For EDCA, the TXOP used is EDCA-TXOP when it is obtained by competing a EDCA contention. The general approach is to use small contention windows (less wait) for high priority flows. There is also a possibility to improve back-off parameters, for example by optimizing the size of contention window $(\mathrm{CW})$ which will be affected by the number of competing nodes [24]. The EDCA protocol uses different $\mathrm{CW}$ sizes for priority differentiation. Enhanced collision avoidance (ECA) scheme is used to improve EDCA protocol under large and more congested network scenarios [25].

2) HCCA: The HCCA is far more complex than PCF with differences of controlled access phase (CAP), in which the hybrid controller manages the traffic flows based on priority polling. HC uses polled-TXOP to manage traffic streams (TS) used to schedule the priorities among nodes. QoS profiling is available for HCCA enabled nodes. Control system design approaches like [26] have also been used for optimization and control of HCCA access scheduling.

\section{QoS provisions in 802.16}

IEEE 802.16 or WiMAX is an evolving set of wireless broadband standards focused on delivering high bandwidth wireless access service to subscribers over the 'last mile'.

QoS is an essential brick of IEEE 802.16 as each connection is associated to a specific QoS. Management messages e.g. dynamic service addition (DSA) and dynamic service change (DSC) are used for maintaining QoS. In WiMAX, the latency on the wireless portion of a network is minimal relative to that on the wired portion of a network.

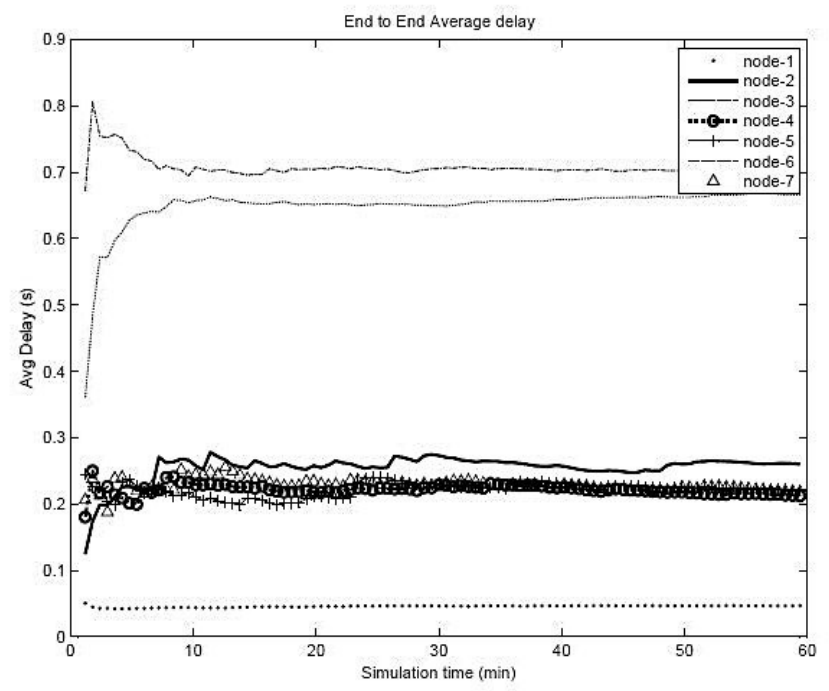

Fig. 5. End to End delay in Wireless Network

\section{Simulation Results}

Two tier Network architecture is simulated in OPNET simulator. A windmill based network (WBN) composed of seven components is simulated. The WBN network uses WLAN for the communication between the individual windmill blocks. The voice, data and video traffic simulated is shown in Table III below. WiMAX is simulated in the tier-2 due to its long range capability and QoS support for the communication between WBN and FBN node. 
TABLE III

TRAFFIC PROFILES

\begin{tabular}{cccc}
\hline Profile & Application & ToS & WiMAX service class profile \\
\hline \hline Gold & Database (Inter-arrival time $=$ exp $(12)$, Trans size $=$ constant(32768)) & BE & UGS, Max sustained traffic- 2Kbps, Max delay = 30ms \\
\hline Silver & Voice $(\mathrm{IP}, \mathrm{G} .729 \mathrm{~A})$ & BE & rtPS, Max sustained traffic- 5Kbps, Max delay = 5ms \\
\hline Bronze & Medium quality video $(10$ frames/sec, 128x120 pixels) & BE & UGS, Max sustained traffic- 2Kbps, Max delay = 20ms \\
\hline \hline
\end{tabular}

Three profiles are used for simulated traffic. The type of service (ToS) is chosen as best effort (BE). It can be seen from the Fig. 5 that node 1 which is configured with 802.11 EDCA takes much less time as compared to DCF and PCF nodes although the WiMAX services are same for all transmissions. This means that QoS flows in the heterogeneous network can be planned for emergency scenarios and therefore near real time support is possible by choosing QoS profiles based on component sensitivity.

\section{CONCLUSION}

In this work, a distributed hierarchic network architecture is proposed for the applications of tele-diagnosis and distant monitoring of a distributed windmill energy generation system. Network simulation with different heterogeneous architectures and protocols is performed to visualize the constraints for long distance communication. In addition, diagnosis tasks at each level of communication need to be allocated for distributed monitoring.

\section{ACKNOWLEDGMENT}

The authors would like to thank OPNET Technologies, Inc. for their licenses under OPNET university program (OUP). A preliminary version of this work was presented in the proceedings of IEEE CCNC 2009 [22] and ICWMC 2009 [2].

\section{REFERENCES}

[1] U. DOE, "Industrial wireless technology for the 21st century," Technology Foresight, 2004/ TF-2004-1., Tech. Rep., 2004.

[2] Z. Khan, D. Catalot, and J. Thiriet, "Hierarchical wireless network architecture for distributed applications," The Fifth International Conference on Wireless and Mobile Communications, Cannes, France, 2009.

[3] A. Mechraoui, Z. H. KHAN, J. Thiriet, and S. Gentil, "Co-design for wireless networked control of an intelligent mobile robot," International Conference on Informatics in Control, Automation and Robotics (ICINCO), Milan, Italy, 2009.

[4] J. Miller, "Vehicle-to-vehicle-to-infrastructure (v2v2i) intelligent transportation system architecture," IEEE 4th Intelligent Vehicles Symposium, Eindhoven, The Netherlands, June 2008.

[5] X. Yang, L. Liu, V. N.H., and F. Zhao, "A vehicle-to-vehicle communication protocol for cooperative collision warning," Mobile and Ubiquitous Systems: Networking and Services, MOBIQUITOUS 04. The First Annual International Conference on, pp. 114- 123, 22-26 Aug. 2004.

[6] D. Gu, P. L. Guangyu, H. Gerla, and M. X. Hong, "Hierarchical routing for multi-layer ad-hoc wireless networks with uavs," Proceedings of 21 st Century Military Communications Conference, MILCOM 2000, Los Angeles, vol. 1, pp. 310-314, October 22-25, 2002.

[7] V. Sarkimaki, R. Tiainen, J. Ahola, and T. Lindh, "Wireless technologies in condition monitoring and remote diagnosis of electric drives; requirements and applications," Power Electronics and App, 2005 European Conference on, pp. 101-105, 11-14 Sept. 2005.

[8] S. Rackley, Wireless Networking Technology: From Principles to Successful Implementation, I. Edition, Ed. Elsevier, 2007.
[9] H. Labiod, H. Afifi, and C. D. Santis, Wi-Fi, Bluetooth, ZigBee and WiMAX, I. Edition, Ed. Springer, 2007. S. Deb, S. Ghoshal, V. Malepati, and D. Kleinman, "Tele-diagnosis: remote monitoring of large-scale systems," in Proc. IEEE Aerospace, vol. 6, 18-25 March 2000, pp. 31-42.

[10] S. Deb, S. Ghoshal, V. Malepati, and D. Kleinman, "Tele-diagnosis: remote monitoring of large-scale systems," in Proc. IEEE Aerospace, vol. 6, 18-25 March 2000, pp. 31-42.

[11] H. Jia, Z. Zhang, P. Cheng, H.-H. Chen, and S. Li, "Study on network selection for next-generation heterogeneous wireless networks," IEEE 17th International Symposium on Personal, Indoor and Mobile Radio Communications, pp. 1-5, 2006.

[12] E. Duarte and T. N. Jr, "A hierarchical adaptive distributed system-level diagnosis algorithm," IEEE Transactions on Computers, vol. 47, pp. 34- 45, Jan. 1998.

[13] A. Subbiah and D. M. Blough, "Distributed diagnosis in dynamic fault environments," IEEE Transactions On Parallel and Distributed Systems, vol. 15, May 2004.

[14] G. Khanna, M. Y. Cheng, P. Varadharajan, S. Bagchi, M. Correia, and P. Verissimo, "Automated rule-based diagnosis through a distributed monitor system," vol. 4, no. 4, pp. 266-279, Oct.-Dec. 2007.

[15] G. Jiroveanu and R. Boel, "A common architecture for distributed diagnosis and wide-area backup protection," in Proc. Eighth IEE international Conference on Developments in Power System Protection, vol. 2, 5-8 April 2004, pp. 756-759.

[16] M. Daigle, X. Koutsoukos, and G. Biswas, "Distributed diagnosis of coupled mobile robots," in Proc. IEEE International Conf on Robotics and Automation ICRA 2006, May 15-19, 2006, pp. 3787-3794.

[17] C. Wang, L. Wang, L. Shi, and Y. Ni, "A survey on wind power technologies in power systems," IEEE Power Engineering Society General Meeting, FL, USA, pp. 1-6, 24-28 June 2007.

[18] E. Hau, Wind Turbines, Fundamentals, Technologies, Application, Economics, 2nd Edition, Ed. Springer, 2006.

[19] T. Burton, D. Sharpe, N. Jenkins, and E. Bossanyi, Wind Energy Handbook. John Wiley \& Sons, 2001.

[20] M. Florkowski and J. Furgal, "Modelling of winding failures identification using the frequency response analysis (fra) method," Journal of Electric Power Systems Research, pp. 1069-1075, 2009.

[21] M. Haffar, J. M. thiriet, and E. Savary, "Modeling of substation architecture implementing IEC 61850 protocol and solving interlocking problems," IFAC conference on field buses, FET 07, France, 2007.

[22] Z. Khan, D. Catalot, and J. Thiriet, "Wireless network architecture for diagnosis and monitoring applications," IEEE-CCNC 09, Las Vegas, USA., 2009.

[23] X. Ma and W. Luo, "The analysis of 6lowpan technology," Pacific Asia Workshop on Computational Intelligence and Industrial Appliaction, IEEE, vol. 1, pp. 963-966, 2008.

[24] T.-C. Tsai and M.-J. Wu, "An analytical model for ieee 802.11e edca," International Conference on Communications, ICC 2005, vol. 5, pp. $3474-3478,2005$.

[25] K. A. Meerja and A. Shami, "Analysis of enhanced collision avoidance scheme proposed for ieee 802.11e- enhanced distributed channel access protocol," IEEE transactions on Mobile Computing, vol. 8, pp. 13531367, 2009.

[26] L. Palopoli, R. Lo Cigno, and A. Colombo, "Control and optimisatin of hcca 802.11e access scheduling," IEEE 46th International Conference on Decision and Control, pp. 4427-4432, 2007. 


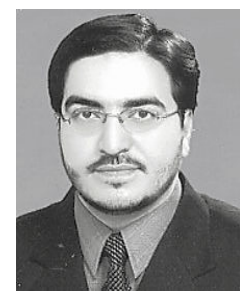

Zeashan H. Khan (S'05) received his Bachelors degree in Electronics Engineering from N.E.D University, Karachi, Pakistan in 2001 and M.S by research degree in Control Systems from INPG, Grenoble, France in 2007. He is currently pursuing his $\mathrm{PhD}$ degree from Joseph Fourier University, Grenoble (GIPSA-Lab UMR 5216 CNRS-INPGUJF). His area of interest includes distributed and cooperative control systems, Remote teleoperation and Co-design of networked control systems (NCS).

$\mathrm{He}$ is a student member of IEEE, IEEE ComSoc, SEE (France) and SNART (Sweden).

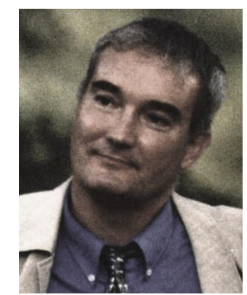

Denis Genon Catalot (M'09) received his B.S. degree in Mathematics and Sciences (1982) from UCB (Lyon), M.S in Electronic Systems (1989) from INPG, Grenoble and PhD in Applied Physics, from Joseph Fourier University, Grenoble, France in 1993. In 1994, he managed the electronics department of the new engineering school at Valence. During the same period he became one of the founder of the research labs in Valence. Since 2008 , he is working as the deputy head of the Networking and Telecommunication Department of Pierre Mendes University at Valence. His work has primarily focused on embedded systems design, networking, low power RF communication systems and system architecture. $\mathrm{He}$ is involved in several industrial partnerships and EU programs. He is a member of the IEEE and EAEEIE.

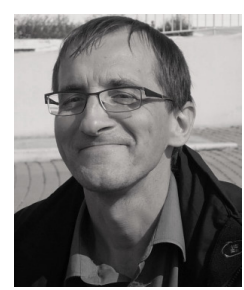

Jean Marc Thiriet (M'01) received his PhD from Nancy University, France. He has been working as a professor in Joseph Fourier University, Grenoble Images Parole Signal Automatique (GIPSA-Lab, UMR 5216, CNRS-INPG-UJF) since September 2005. Previously, he was an associate professor at Henri Poincar University, Nancy 1, Centre de Recherche en Automatique de Nancy, France. His research activities involve dependability of the networked distributed control systems and codesign of networked systems for diagnosis. He is currently serving as Head of the Control System Department at GIPSA-Lab, Grenoble 